\title{
Thymoma developing 2 years after induction of immunosuppressive therapy for ocular myasthenia gravis
}

\author{
Takashi Iwata ${ }^{1}$, Kantaro Hara ${ }^{1}$, Hidetoshi Inoue ${ }^{1}$, Aya Yamamoto ${ }^{1}$ and Osamu Mimura ${ }^{2}$ \\ ${ }^{1}$ Department of Thoracic and Cardiovascular Surgery, Kansai Rosai Hospital, 3-1-69 Inabaso, Amagasaki, Hyogo, 650-8511 Japan \\ ${ }^{2}$ Department of Ophthalmology, Hyogo Medical College, 1-1 Mukogawa-cho, Nishinomiya, Hyogo, 663-8501 Japan
}

\begin{abstract}
A 73-year-old woman with myasthenia gravis who had been treated by prednisolone and tacrolimus for 23 months, was referred to our hospital for acute chest pain. Chest computed tomography (CT) demonstrated an anterior mediastinal mass of $6 \mathrm{~cm}$ in size, approximately. Chest CT that was taken at the time of diagnosis of myasthenia did not show any evidence of an anterior mediastinal mass. Extended thymectomy with combined resection of the lung and the pericardium was performed a month later. Pathology disclosed type B3 thymoma. The postoperative course was uneventful and the patient was discharged on the day 14 . Any evidence of recurrence of the thymoma is not found 39 months after the surgery.
\end{abstract}

\section{Introduction}

Development of myasthenia gravis (MG) after thymectomy is rare but sometimes occurs[1,2]. However, thymoma arising years after induction of drug therapy for MG is extremely rare and such case has never been reported in the literature to our knowledge. Here in, we report a case of type B3 thymoma occurred 2 years after induction of immunosuppressive therapy for ocular myasthenia, successfully treated by extended thymectomy and combined resection of surrounding invaded organs, and following radiotherapy.

\section{Case report}

A 73-year-old woman with MG who had been treated by $3 \mathrm{mg}$ of prednisolone and $3 \mathrm{mg}$ of tacrolimus a day, was referred to the department of cardiovascular medicine at our hospital, for acute chest pain that was exacerbating on deep breathing. This was the second consult for her to our hospital because of chest pain. She had been diagnosed with ocular myasthenia for ptosis 23 months ago, because the results of the Tensilon ${ }^{\oplus}$ test and the ice pack test were both positive. Serum level of anti-acetylcholine receptor (AChR) antibody titer was $3.5 \mathrm{nmol} / \mathrm{L}$ at that time. Chest computed tomography (CT) at the time of diagnosis did not show any evidence of an anterior mediastinal mass (Figure 1A). As an initial treatment, pyridostigmine bromide was inducted however it was not effective at all. Thus, immunosuppressive therapy was inducted with $30 \mathrm{mg}$ of prednisolone every second day. This was effective to alleviate the ptosis but the patient got diabetic. Therefore, prednisolone had been gradually decreased in combination of tacrolimus administration since 20 months ago. The patient experienced anterior chest pain 9 month after the initiation of the treatment. She presented to the department of cardiovascular medicine at our hospital for the first time. Chest CT taken at that time did not demonstrate any evidence of a mediastinal mass again, despite a small irregularity on the surface of the mediastinal pleura (Figure 1B). Angina was suspected but the patient did not received further evaluation and treatment because the pain was relieved spontaneously.

At the time of the second visit, recurrence of angina was suspected again. Coronary angiography was emergently performed but did not show any ischemic findings. Chest CT demonstrated an anterior mediastinal mass of $6 \mathrm{~cm}$ in size, approximately (Figure 1C). Then the patient was referred to our department for further evaluation and treatment. All results of blood chemical studies were within normal ranges, otherwise serum level of anti-AChR antibody titer $(1.2 \mathrm{nmol} / \mathrm{L})$ and serum level of carcinoembryonic antigen $(12.7 \mathrm{ng} / \mathrm{mL})$. The doses of prednisolone and tacrolimus were decreased to $2 \mathrm{mg} /$ day and $2 \mathrm{mg} /$ day preoperatively for a month, respectively, and then extended thymectomy was performed. The tumor infiltration to the surrounding organs was observed intraoperatively, thus combined resection of pericardium, right lung, and right phrenic nerve, and the plication of the right diaphragm were added. The postoperative course was uneventful and the patient was discharged on the day 14 .

Pathology demonstrated that the tumor was consisted with proliferation of polygonal or round cells forming solid nests. Atypia, keratinization, and intercellular bridges were not observed. CD1apositive lymphocytes were scattered focally in the tumor tissue. Immunohistochemical study evidenced negative stain of CD5, CD177, Bcl-2, chromogranin A, synaptophysin, and CD56. AE1/ AE3 were positive and EMA was partially-positive. Type B3 thymoma was thus diagnosed. Infiltration into the lung and pericardium was microscopically demonstrated. Therefore, irradiation therapy (40 Gy/20 fraction) was added since 5 weeks after the operation.

The doses of immunosuppressive agents could be gradually less decreased postoperatively. She was taking $2 \mathrm{mg}$ of tacrolimus alone and prednisolone was ceased 3 months after the operation, and did

Correspondence to: Takashi Iwata, Department of Thoracic and Cardiovascular Surgery, Kansai Rosai Hospital, 3-1-69 Inabaso, Amagasaki, Hyogo, 650-8511 Japan, Tel:+81-6-6416-1221; Fax: +81-6-6419-1870; E-mail: iwata-takashi@ kansaih.johas.go.jp

Key words: thymoma, mediastinal tumor, surgery, myasthenia gravis

Received: August 04, 2017; Accepted: September 01, 2017; Published: September 04, 2017 


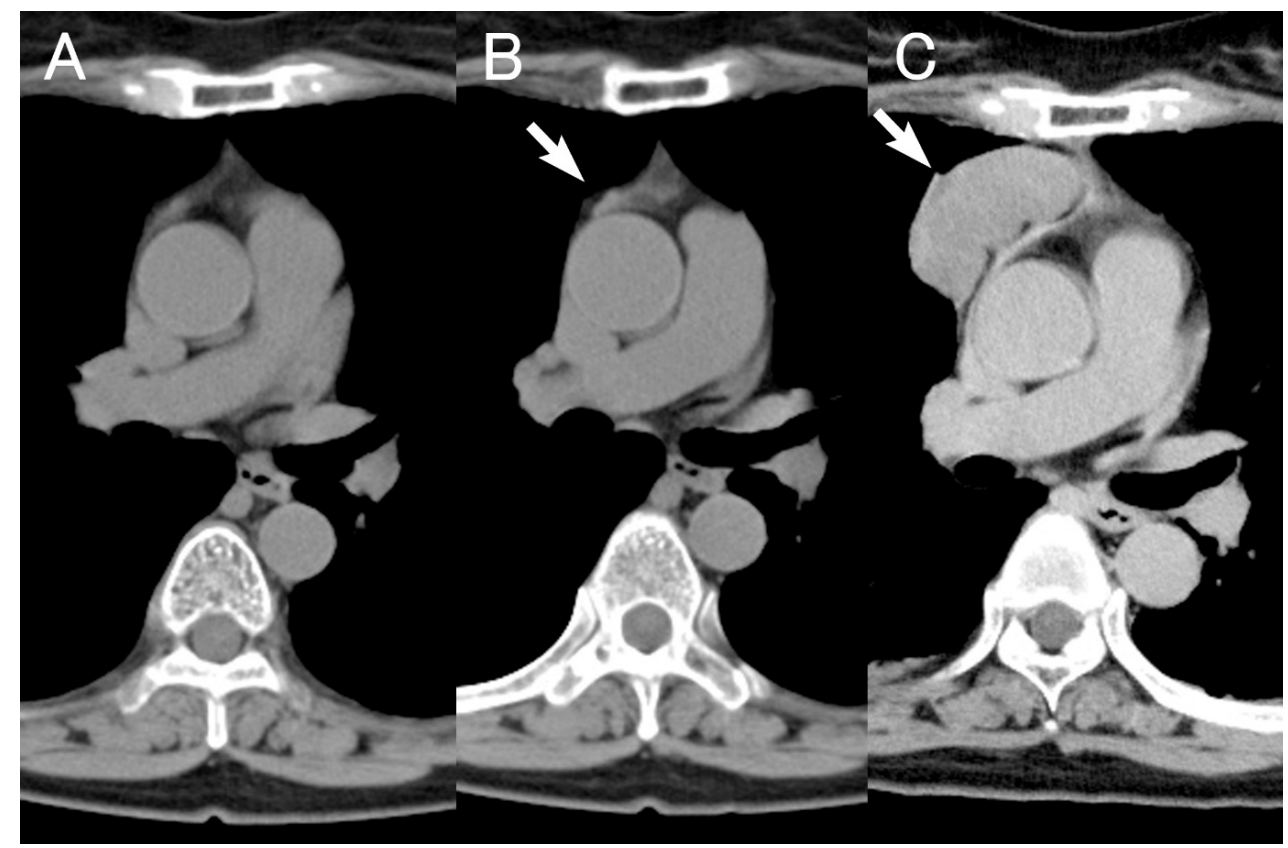

Figure 1. A. Chest computed tomography (CT) taken at the time of diagnosis of ocular myasthenia doesn't demonstrate any findings of an anterior mediastinal mass. B. Chest CT taken 9 months after the induction of treatment for myasthenia also doesn't show any abnormal finding despite a small irregularity on the surface of the mediastinal pleura (arrow). C. Chest CT taken 2 years after induction of treatment for myasthenia shows a round shaped mass in the anterior mediastinum, of approximately $6 \mathrm{~cm}$ in size (arrow)

not experience the ptosis at all. Serum level of anti-AChR antibody titer remained $1.7 \mathrm{nmol} / \mathrm{L}$ and the serum level of carcinoembryonic antigen was decreased to $8.6 \mathrm{ng} / \mathrm{mL}$. Any evidence of recurrence of the thymoma is not found 39 months after the surgery.

\section{Discussion}

Chest CT is usually taken before starting the treatment for MG because the patients with MG is frequently accompanying with thymomas. If the patient has thymoma, thymectomy is indicated. If without thymoma, thymectomy is usually not suggested for patients with ocular myasthenia. On the other hand, for those with systemic myasthenia, combination of thymectomy and drug therapy is recommended. In the presented case, chest CT taken before induction of drug therapy did not demonstrate any evidence of an anterior mediastinal mass. It doesn't mean that there was truly no tumor lesion in the thymus. There might have been some subclinical "microscopic" thymoma already in the thymus that could not be visualized by usual chest CT. Microscopic thymoma is rare entity but reported in the literature, that is incidentally discovered in the specimen taken by thymectomy that had been performed in patients with myasthenia gravis or pure red cell aplasia, even without any obvious finding of thymoma in preoperative CT $[3,4]$.

In this case, after failure of pyridostigmine monotherapy, immunosuppressive therapy using corticosteroid was inducted to alleviate the myasthenic symptom. Corticosteroid itself is well-known to shrink thymomas. We inferred that this corticosteroid therapy might have been suppressing the tumor growth for a length of time, until the patient got diabetic. According to the decrease of the steroid dose in treatment of diabetic status, the tumor was gradually growing at accelerated pace, and finally grew enough to raise anterior chest pain. Actually, during 2 years since diagnosis of myasthenia, administrated dose of prednisolone had been decreased $30 \mathrm{mg} / 2$ days to $3 \mathrm{mg} /$ day, gradually. However, in order to get tumor shrinkage, method of corticosteroid administration is usually as pulse therapy and the given dose is much more[5]. We assume that the thymoma of this case might have been extremely sensitive to corticosteroid. Even the given starting dose was low but would have been enough to suppress the tumor growth. The gradual decrease of corticosteroid might have given a drastic spurt of tumor growth during the 2 years.

The existence of microscopic thymoma in patients with ocular thymoma is another issue in this case. If the incidence of microscopic thymoma were frequent in patients with ocular myasthenia, the development of thymoma after starting of drug therapy would be more frequent. We do not have much data about the frequency of microscopic thymoma in patients with ocular myasthenia, however, periodical chest radiograph or CT would be needed even if the patient were without thymoma at the beginning of the treatment.

\section{Conclusion}

Even in patients with ocular myasthenia that initially had no thymomas, careful check up for newly developing thymoma could be important, as well as the treatment of myasthenic symptoms.

\section{Acknowledgements}

We thank Drs. Shin'ichi Nakatsuka, Teruaki Nagano, and Takayoshi Goto for their contribution in pathological diagnosis.

\section{Disclosure statement}

This study was funded by Kansai Rosai Hospital. The all authors have no conflict of interest.

\section{References}

1. Sun XG, Wang YL, Liu YH, Zhang N, Yin XL, et al. (2011) Myasthenia gravis appearing after thymectomy. J Clin Neurosci 18: 57-60. [Crossref]

2. Kondo K1, Monden Y (2005) Myasthenia gravis appearing after thymectomy for thymoma. Eur J Cardiothorac Surg 28: 22-25. [Crossref]

3. Chalabreysse L, Orsini A, Vial C, Tronc F (2007) Microscopic thymoma. Interact Cardiovasc Thorac Surg 6: 133-135. [Crossref] 
Iwata T (2017) Thymoma developing 2 years after induction of immunosuppressive therapy for ocular myasthenia gravis

4. Cheuk W, Tsang WY, Chan JK. (2005) Microthymoma: Definition of the entity and distinction from nodular hyperplasia of the thymic epithelium (so-called microscopic thymoma). Am J Surg Pathol 29: 415-419. [Crossref]
5. Kobayashi Y, Fujii Y, Yano M, Sasaki H, Yukiue H, et al. (2006) Preoperative steroid pulse therapy for invasive thymoma: clinical experience and mechanism of action. Cancer 106: 1901-1907. [Crossref]

Copyright: (C2017 Iwata T. This is an open-access article distributed under the terms of the Creative Commons Attribution License, which permits unrestricted use, distribution, and reproduction in any medium, provided the original author and source are credited. 